\title{
A Virtual Learning Environment for Real-World Networking
}

\author{
Bruce Kneale and llona Box \\ University of Western Sydney, Penrith, Australia
}

\section{b.kneale@uws.edu.au i.box@uws.edu.au}

\begin{abstract}
Virtual learning environments are a solution to some of the problems of providing an authentic learning environment. We encountered problems such as lack of funding and physical space, and risks and threats to our network environment when we contemplated providing a real, physical specialist laboratory to teach computer networking. We solved most of our problems by developing Velnet, a virtual environment for learning networking. Velnet consists of one or more host machines and operating systems, commercial virtual machine software, virtual machines and their operating systems, a virtual network connecting the virtual machines, and remote desktop display software. Our first experiment with Velnet was in a standalone configuration, without remote desktop display. The initial pilot had students connecting to Velnet via our institution's network. Velnet performed well under this restricted access environment. We are developing a virtual reality overlay of Velnet to be able to present computernetworking concepts. We are also investigating the changes we can make to our instructional design and assessment strategies, and the consequent learning experiences of the students.
\end{abstract}

Keywords : virtual learning environment, Velnet, computer networking

\section{Introduction}

It is widely recognised that learning about computer networking requires a 'hands on', authentic learning experience (Agilent Technologies, 2001; Asia Computer Weekly, 1999; Costa, 2000; Kasim, 2002; Logical UK Ltd, 2002; Mateyaschuk, 1999; Montgomery, 2002; Nortel Networks, 2002). Our experiences tend to support this requirement. However, being able to use an authentic learning environment and incorporate authentic and valid assessment tasks can be problematic. The problems we encountered included: 1) lack of funds to establish and maintain networking hardware and software in sufficient quantity so that each student can have equal access to the learning environment inside and outside scheduled classes; 2) lack of physical space, both air-conditioned space for servers, and for the hardware used directly by the students; and 3) lack of a secure network environment that can reliably protect the other services provided on the same machines or network.

This paper details an attempt to provide a secure, cost-effective approach to 'hands-on' networking using a virtual learning environment. We describe the design of Velnet, a virtual environment for learning networking. Velnet uses existing hardware and software to present a virtual network environment in which students can securely and safely learn about and experiment with computer networking technologies. The initial development of Velnet is complete and has been piloted. It was found that our problems

Material published as part of these proceedings, either on-line or in print, is copyrighted by Informing Science. Permission to make digital or paper copy of part or all of these works for personal or classroom use is granted without fee provided that the copies are not made or distributed for profit or commercial advantage AND that copies 1) bear this notice in full and 2) give the full citation on the first page. It is permissible to abstract these works so long as credit is given. To copy in all other cases or to republish or to post on a server or to redistribute to lists requires specific permission from the publisher at Publisher@InformingScience.org with providing an authentic learning environment have been largely overcome.

Velnet has provided us with the opportunity to reconsider our instructional design and assessment strategies. We discuss these and areas for further research. 


\section{Experiences and Motivation}

The first author taught two computer networking subjects during 2002, one covering fundamental concepts and the other covering topics that are more advanced. Both courses included practical components. The students had two hours per week in an existing data communications laboratory and were required to complete a set of practical exercises. It was apparent that the learning outcomes from the practical exercises had to be compromised to guarantee all students would be able to complete the exercises in the allotted time. To accommodate this compromise the practical exercises were designed as simple, taskoriented, and targeted the slowest student.

The practical exercises were simplified as it was felt that two hours per week did not allow complex exercises to be attempted. With a week between lab sessions, it was difficult to design exercises to run over multiple lab sessions and maintain the continuity of the learning experience.

The practical exercises were task-oriented by giving each student a step-wise task list that could be completed in the allotted time.

The practical exercises were targeted at the slowest working student. The exercises were an assessable component, contributing to a final grade. So that each student had an equal opportunity to attempt and complete the exercises, they were restricted to an estimate of what a student who is capable of just a passing grade could accomplish in two hours.

Many students were working through the task lists without any real understanding of what they were doing, demonstrating that they were engaged in surface learning. High achieving students were not challenged by the exercises as they were completing exercises in minimal time and gaining no sense of accomplishment due to the relative ease and simplicity of the tasks.

It was felt that if students had additional time to prepare for and extend exercises, then exercises that are more challenging could be designed. Three essential requirements were identified to allow this: 1) Laboratories should be available to students outside of the scheduled laboratories of two hours per week; 2) Students should have the facility to start, pause, and continue an exercise over a period of time; and 3) Access to the learning environment should be safe, secure and available 24 hours a day, seven days a week.

These requirements were not met by the existing facilities and policies. Specialist laboratories, such as the data communications laboratories, must be supervised. Consequently, laboratories are only available to students during scheduled, supervised sessions. Laboratories are used for many courses, each course requiring a particular configuration. Therefore exercises could not be carried over to the next week's class, as the configuration does not save each student's progress on a task.

It was felt that a learning environment that students could access remotely would meet these requirements. Initial investigations focused on a physical network system that could be accessed remotely but several problems were identified.

\section{Problems with Providing a Physical Network}

\section{Lack of Funds}

To provide a fully equipped networking laboratory: cabling, hub equipment, firewalls, servers, workstations, etc. can be costly. Typically, we have 20 students per laboratory class. To provide 20 work areas so that each student has equal access to the equipment during class would cost about AU\$160,000. Since we cannot provide such equipment for the exclusive use of an individual, we form the students into groups. Students do not always work well in groups, with some students doing the work while others merely watch. When it comes to assessment it is pen and paper based and lacks authenticity. 
The lack of funds to establish and maintain networking hardware and software in sufficient quantity so that each student can have equal access to the learning environment inside and outside scheduled classes is perhaps common to many institutions. One means of overcoming this problem is for the institution to form an alliance with an industry partner. An example is the Cisco Networking Academy.

Cisco Systems, the world's biggest data networking company (Cane, 1999) has a standardized technology curriculum presented in 145 countries (Cisco, 2002). Some institutions have incorporated the Cisco curriculum into their degrees, while others have used it as a value-added product, a student being able to graduate with a degree and Cisco certification. The incorporation of the Cisco curriculum by many institutions is favoured by some and disdained by others. Those that favour the Cisco academy approach see it as an opportunity to have curriculum and teaching resources freely available, even though there are training and equipment costs (approximately a minimum of AU\$25,000 for our institution to start up a value-added course). Those that disapprove see the possibility of diminishing returns on investment as more and more institutions join the academy, reducing each institution's market share of paying students. The disapproving also sense a risk of committing to a company in an industry that is notoriously unstable, the subsequent replenishment of lost non-corporate, academic skills, such as curriculum and teaching material development, being an issue. There are also issues related to proprietary bias and whether institutions see this as limiting their students.

Another solution to overcome the lack of funds, and the direction we chose, was to develop a virtual learning environment. The cost of Velnet to provide the equivalent to a fully equipped laboratory of AU\$160,000 is approximately AU\$55,000, one third of a fully equipped laboratory. The ongoing costs of running a fully equipped laboratory are significant. Velnet also costs less to run as it can be cloned to other hosts and does not have the overheads of a fully equipped laboratory such as air cond itioning.

The use of virtual reality to save money is not new. The use of simulations in industry for research and development "has blossomed since the proliferation of desktop personal computers, windows user environment, and modular software development techniques" (Harrold, 2000). Harrold goes further by providing a number of examples of simulations used for analysis, design, debugging and training that have saved money.

\section{Lack of Physical Space}

A fully equipped laboratory would require more than one room. The equipment needs to be housed in a temperature-controlled environment of about $800 \mathrm{~m}^{2}$. Velnet can be housed in less than $200 \mathrm{~m}^{2}$; less than one quarter of a fully equipped laboratory and; does not need to be temperature controlled, as the heat generated is less, as fewer devices are required. Physical rooms, at our institution, are always in high demand and short supply. Making more efficient use of the available space influenced our choice to develop a virtual rather than real learning environment.

\section{Lack of a Secure and Manageable Networking Environment}

An average Australian undergraduate combines around 15 hours per week of paid work with full-time study (McInnis \& Hartley, 2002). Though we would like to have students consider study as their first priority, the reality is it is not the case; to expect students to be in laboratories "practicing" on campus is unrealistic. We can accommodate these realities of student life by providing safe and manageable learning environments for students, academics, and technical staff.

A segregated learning environment, such as a specialist laboratory, can solve the security concerns often raised by technical staff (Davis, Ransbottom, \& Hamilton, 1998). However, it does not address the problem of safe access to facilities nor does it deal with management issues such as the 'run- up' and 'teardown' time needed for each laboratory class. 
Students need to "practice" their networking skills (Marti, Pooch, \& Hamilton, 1996) but the amount of time a student can spend in the laboratory is limited by the number of hours that the laboratory is available each week divided by the total number of partic ipating students.

Velnet addresses the security concerns raised by technical staff by isolating the virtual devices from the outside world. Velnet can address the accessibility issues by providing remote access for the student, effectively making the laboratory operating hours to twenty-four hours a day, seven days a week.

The issue of 'run-up' and 'tear-down' can be addressed by maintaining multiple 'ready-to-go' configurations. A student can choose the configuration for a particular task when using the virtual environment.

\section{Virtual Learning Environments and Virtual Reality}

Winn and Jackson (Winn \& Jackson, 1999) presented 14 propositions about the use of virtual reality in learning environments. Those applicable to our situation include:

1) Virtual environments (VE) are cheaper than a physical environment. Winn and Jackson go further by proposing that though a virtual environment may be less real, the trade-off with cost does not make the outcomes poorer. It may be that skills are more easily transferred because the virtual environment is lower fidelity. A student expecting from a high fidelity VE that the real world will be the same is confused by even small changes. Lower fidelity VE make the student generalise what they have learnt in the real world.

2) Virtual environments are safer. The VE provide a safe place to practice risky tasks. In our situation the safety of the network is one concern. Another is that the students feel safe to experiment without causing damage for which they may feel they would be reprimanded. This was also argued by Forbus $e t$ al. (Forbus et al., 1999)

3) Natural interaction with the VE allows students to experience metaphorical concepts and undetectable phenomena. Students would apply their networking skills and knowledge in a real environment by using a keyboard, a mouse, and a monitor. Velnet emulates this natural interaction. Taking this proposition further, an overlay of Velnet plus a VE helmet and glove would put the students in a VE where they could become part of the network from a bit on a bus, to a bridge, server or firewall; time can be slowed; concepts and non-detectable physical forms can take shape (Winn and Jackson refer to this as reification).

4) There is growing evidence that students, particularly those not academically inclined or committed, such as the majority of students undertaking higher education (Biggs, 1999), are more likely to do well and develop familiarity with the subject material in a VE. The students also then see the VE as valuable. Providing authentic learning experiences by modifying the laboratory environment may increase student engagement in the learning process and lead to high-order thinking in students (Cruikshank, 2002).

5) Constructivist concepts of learning and "first-hand" experience (Clancey, 1993) allows students to take what is familiar to them and add to this knowledge about networking that they gain by experiencing the VE. The VE can then be used to assess the student in an activity where the student demonstrates their construction of meaning given a problem to solve. Such an authentic assessment being judged on the student's holistic performance of the tasks (Montgomery, 2002). This proposition is also lent weight by the advice from Braathen and Robles (Braathen \& Robles, 2000).

6) The VE situates learning in a real context. The appearance of each virtual machine in Velnet is as it would appear as if a separate physical machine. Velnet has presence; the students can be convinced that they are working with a real network. They would directly connect their experience with Velnet to the real situation. Velnet is "real" enough for common networking tools such as protocol analysers and ne twork management software to be employed to solve real problems. Students will encounter the same 
challenges using Velnet as in the real world: idiosyncratic operating systems, buggy implementations of network software, and difficult to understand user docume ntation.

Velnet's virtual environment can be extended. A medium-term goal for the Velnet project is to provide 24 hours a day, seven days a week access to Velnet via the Internet. Though there are arguments for and against such a goal (O'Donoghue, Singh, \& Dorward, 2001; Piccoli, Rami, \& Blake, 2001), with careful instructional and assessment design the benefits expected and, arguably, the inevitability of the role the Internet will play in course delivery make this goal probable.

The long-term future of Velnet may be in the ability to convert what is a good improvement on our previous situation into an articulate virtual laboratory (AVL) (Forbus et al., 1999). An AVL, according to Forbus et al. would address problems of marking, timely feedback, coaching, and guidance.

\section{The Components of Velnet}

The components of Velnet, shown in Figure 1, are: the host machine, a personal computer; the host machine's operating system; VMWare ${ }^{\mathrm{TM}}$, commercial virtual machine software; virtual machines and their operating systems; a virtual network connecting the virtual machines, and; VNC, Virtual Network Computing software (Richardson, Stafford-Fraser, Wood, \& Hopper, 1998). Velnet appears as several windows on the host machine's desktop (Figure 2).

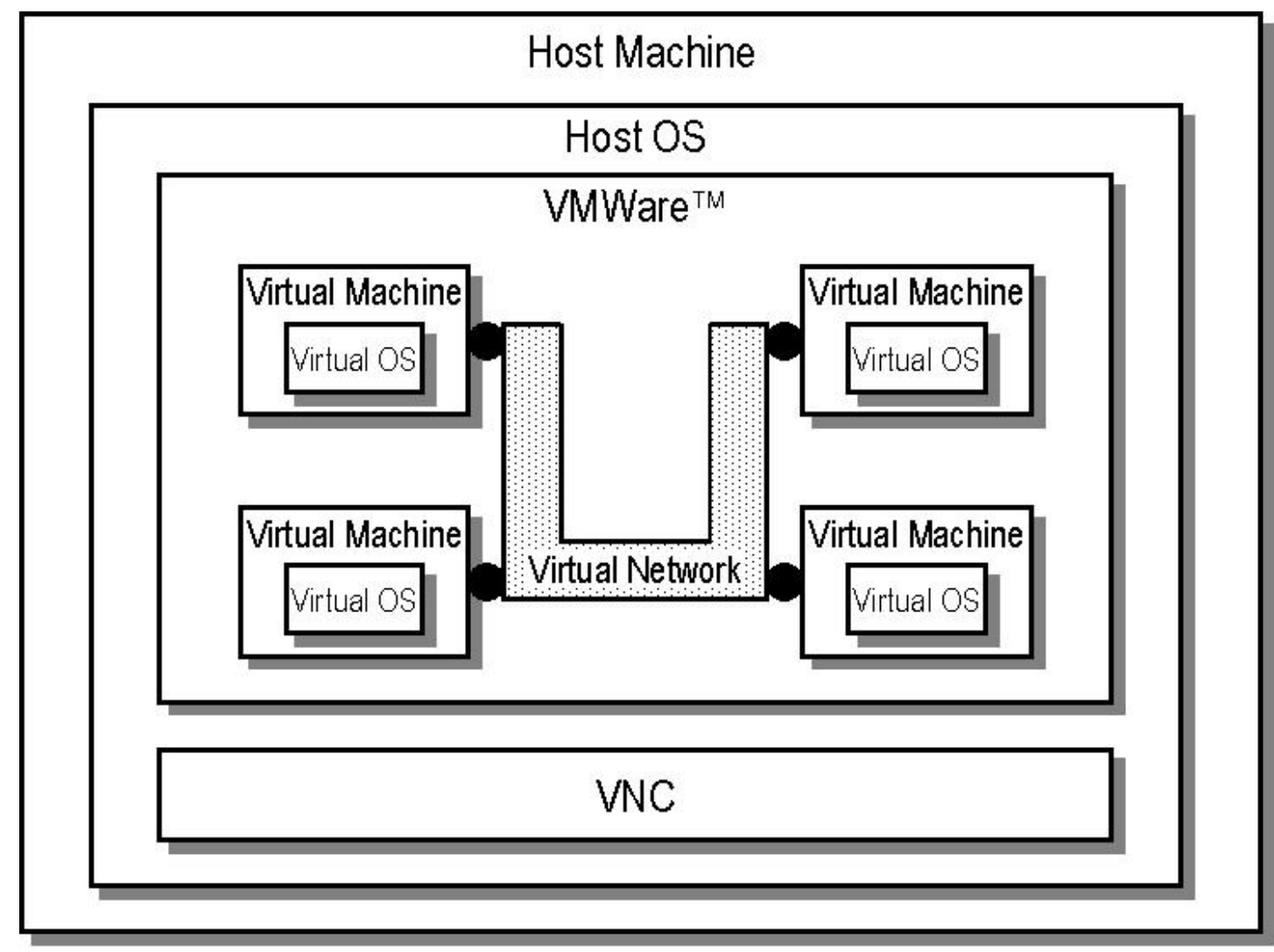

Figure 1. The components and configuration of Velnet

\section{Host Machine}

Velnet is designed to operate on a single desktop computer. The number of virtual machines that can "exist" simultaneously on a host computer depends on the amount of memory and the CPU speed. A host computer will be emulating several virtual machines. The hardware specification used for the initial 
development of Velnet was: CPU, AMD Athlon ${ }^{\mathrm{TM}}$ 1800+; 1GB of memory (RAM); 60GB hard disk, and; an Intel® Pro 100+ network interface card.

The amount of memory (RAM) required on the host machine is calculated based on the memory requirements of the host operating system plus the memory requirements of the virtual operating systems that are running concurrently. For example, a configuration of three virtual machines with, say, Linux ${ }^{\circledR}$ on two virtual machines and Windows NT 4 (WS) on the third, and a host running Windows 2000 would be calculated as:

$\begin{array}{llr}\text { Host }- \text { Windows } 2000 \times 1 \times 128 \mathrm{MB} & = & 128 \mathrm{MB} \\ \text { Virtual }- \text { Linux }{ }^{\circledR} \times 1 \times 128 \mathrm{MB} & = & 256 \mathrm{MB} \\ \text { Virtual }- \text { Windows NT } 4 \text { (WS) } \times 1 \times 32 \mathrm{MB} & = & 32 \mathrm{MB} \\ \text { Minimum Total Host Memory Required } & = & 416 \mathrm{MB}\end{array}$

The required disk capacity on the host machine is calculated based on the storage requirements of each operating system whether or not any virtual machines are running. For example, a configuration of five virtual machines with, say, Linux ${ }^{\circledR}$ on three virtual machines and Windows NT 4 (WS) on another two, (even though no virtual machines may be running) and a host running Windows 2000 would be calc ulated as:

$\begin{array}{llr}\text { Host }- \text { Windows } 2000 \times 1 \times 4 \mathrm{~GB} & = & 4 \mathrm{~GB} \\ \text { Virtual }- \text { Linux } ® \text { × } 3 \text { × } 4 \mathrm{~GB} & = & 12 \mathrm{~GB} \\ \text { Virtual - Windows NT } 4 \text { (WS) } \times 2 \times 1 \mathrm{~GB} & = & 2 \mathrm{~GB} \\ \text { Minimum Total Host Disk Capacity Required } & = & 18 \mathrm{~GB}\end{array}$

The first experiment with Velnet had one host computer running Windows ${ }^{\circledR} 2000$ with five virtual ma-

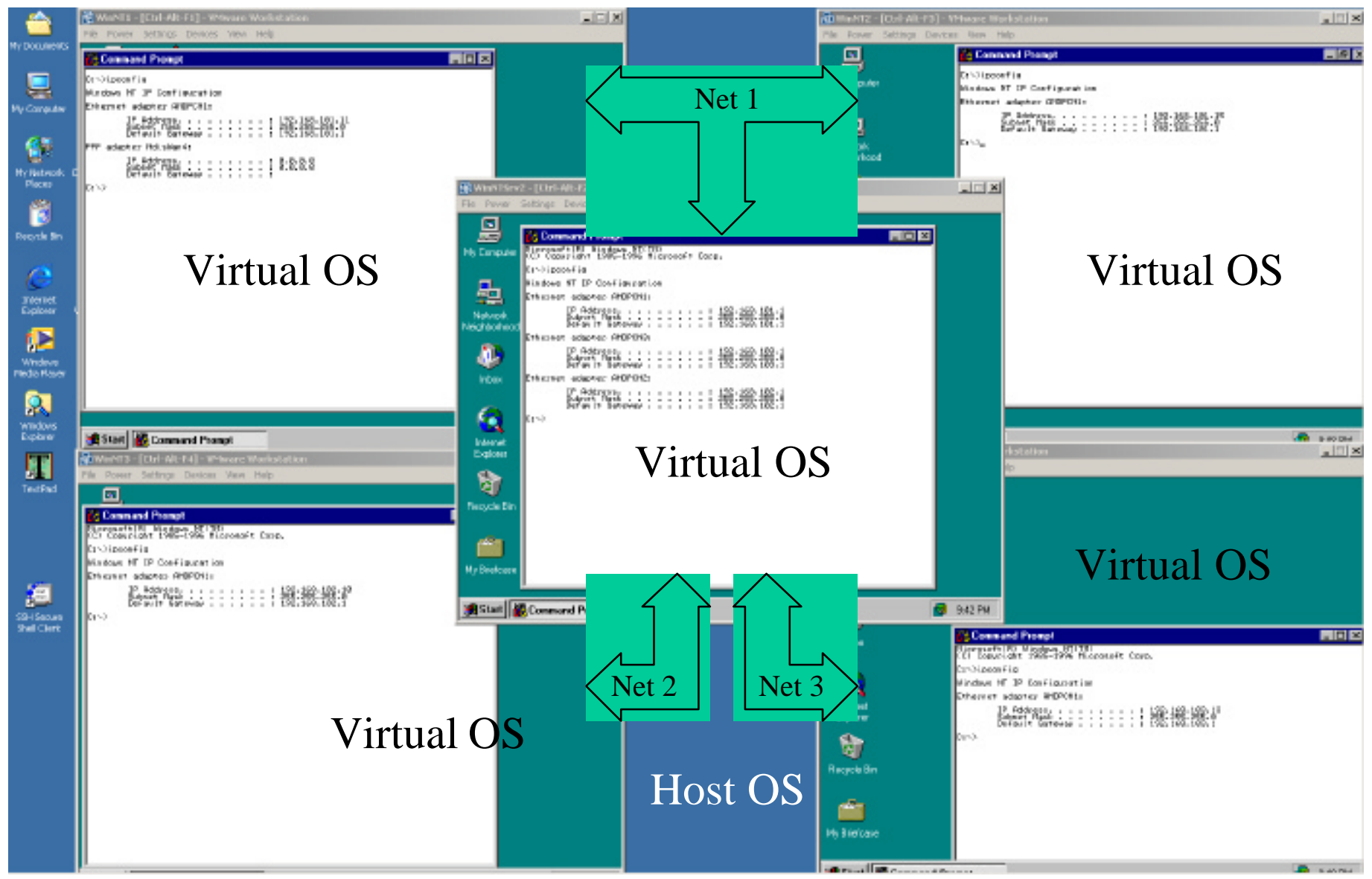

Figure 2. A Windows 2000 host with five virtual machines, each running Windows NT 
chines. Four of the virtual machines ran Windows ${ }^{\circledR}$ NT 4 Workstation and the fifth virtual machine ran Windows NT® 4 Server that acted as a routing device for the other virtual machines. The Velnet pilot used six host machines running Windows ${ }^{\circledR} \mathrm{XP}$, each with three virtual machines. One virtual machine ran OpenBSD while the other two virtual machines ran Windows ${ }^{\circledR}$ NT Workstation. Windows $®$ XP remote desktop tools were used to provide students with remote connectivity.

\section{Host Operating System (Host OS)}

Windows ${ }^{\circledR} 2000$ was initially chosen as the operating system for the host computer for ease of configuration and student familiarity with the system. Difficulties were encountered when using VMWare ${ }^{\mathrm{TM}}$ and VNC, the two applications contended for mouse pointer control when used with virtual machines running GUI based operating systems. The problem was not apparent for virtual machines running text based operating systems such as OpenBSD.

Linux® cannot be used as a host operating system in conjunction with remote access to Velnet as VMWare $^{\mathrm{TM}}$ uses Xwindows graphics extensions which are only available in a standalone configuration.

\section{VMWare ${ }^{\mathrm{TM}}$}

Velnet requires software that provides multiple hardware environments on the one host computer. Opensource applications that provide virtual PC hardware were not generally available. Two commercial products were reviewed for applicability to this project: VMWare ${ }^{\mathrm{TM}}$ (VMWare ${ }^{\mathrm{TM}}$ Workstation, 2002) and Virtual PC'M (Virtual PC'M , 2002).

Both applications provide virtual Intel ${ }^{\circledR}$ x86 hardware environments that are compatible with various host operating systems. While both applications provide virtual hardware environments, the desired network configuration for teaching purposes required connection of two virtual machines via a virtual serial line. At the time of writing, VMWare ${ }^{\mathrm{TM}}$ was the only product that provided the capability to connect two virtual machines via a virtual serial line (VMWare ${ }^{\mathrm{TM}}$, 2002). Network configurations that do not need serial lines may be acceptable for use with Virtual PC ${ }^{\text {TM }}$ (Connectix, 2002).

\section{Virtual Machine}

The virtual machine emulates an Intel ${ }^{8}$ x86 hardware environment. From the time of "switching on" the machine it appears as if it were a real, physical computer (Figure 3).

\section{Virtual Operating System (Virtual OS)}

The choice of operating system depends on desired learning outcomes, cost and memory available in the host machine. Even though there is one host machine, each virtual machine has a separate identity and as such, appropriate licensing must be obtained for any operating system used.

The choice of virtual machines for the initial standalone experiment was Windows® NT Workstation and Windows ${ }^{\circledR}$ NT Server. The choice of virtual machines for the pilot was OpenBSD and Windows ${ }^{\circledR}$ NT Workstation.

Judicious use of open source operating systems such as Linux ${ }^{\circledR}$ and OpenBSD provides less expensive alternatives.

\section{Virtual Network}

The virtual network runs in VMWare ${ }^{\mathrm{TM}}$, as a function of this application. Some of the components of a physical network such as switches and network adapters are emulated in the virtual network. Routing is supported by operating systems, which have this function and are installed on the virtual machines. 


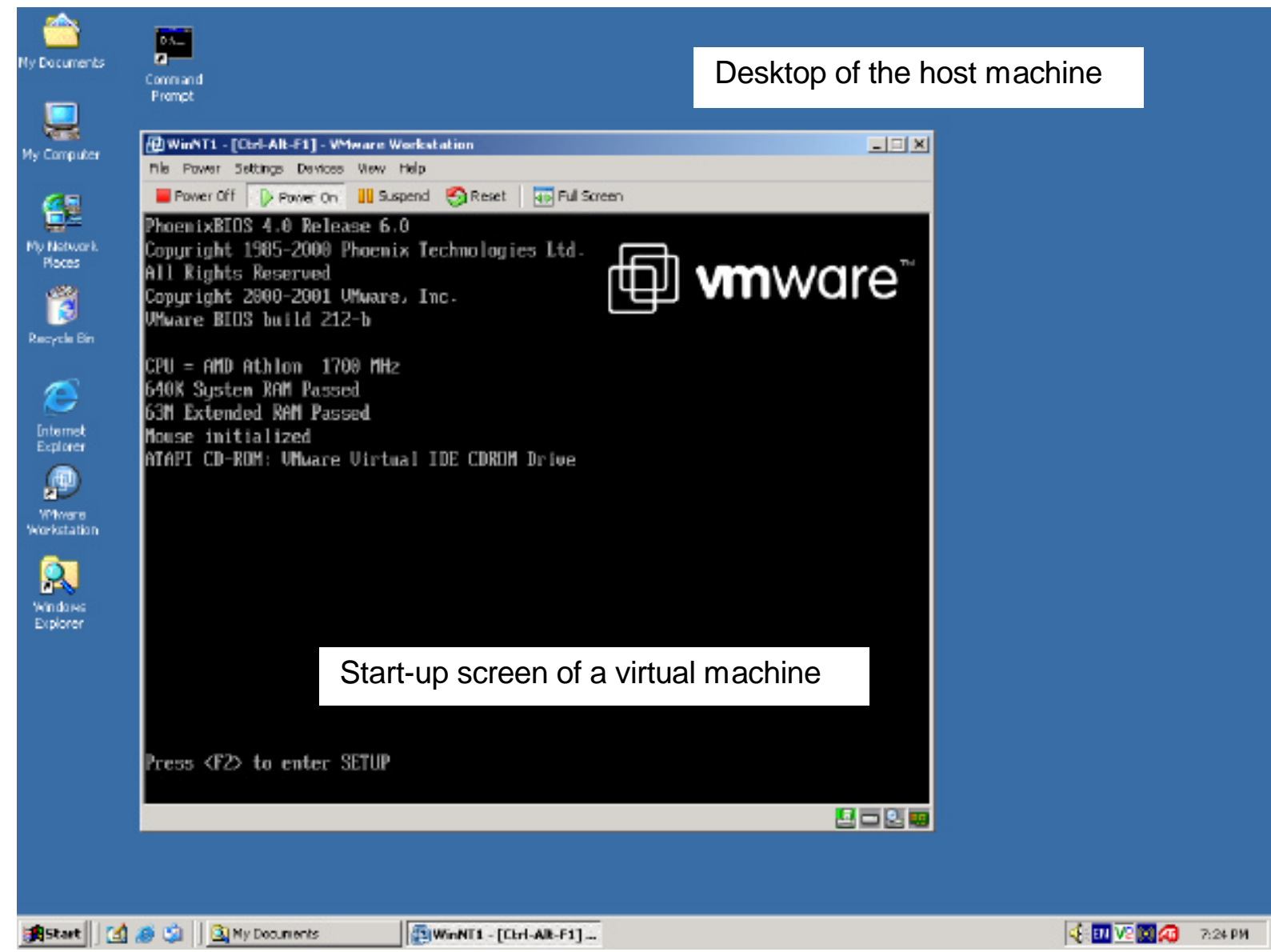

Figure 3. The start up of a virtual machine appears the same as a physical computer.

\section{Remote Desktop Display (RDD)}

VNC is a remote display system which allows students to remotely control the 'desktop' of the Velnet host machine from anywhere on the Internet. VNC is a client/server system; the server portion runs on the host machine. The client portion of the system runs on any machine from which a student wishes to remotely control the host machine's desktop.

Remote Desktop for Windows@ XP is similar in functionality to VNC except the server component is built into the Windows ${ }^{\circledR}$ XP operating system. Microsoft ${ }^{\circledR}$ supplies client software for most Windows ${ }^{\circledR}$ operating systems and certain Apple ${ }^{\circledR}$ operating systems.

\section{Connecting to Ve Inet}

Remote display software allows students to interact with the virtual network from anywhere on the Internet, although for the purposes of this experiment, access was restricted to client computers within the institution's network. A typical remote access session to Velnet is shown in Figure 4. In Figure 4, Velnet is running as six host machines, each representing an individual network (a) and students gain remote access via any computer (b) connected to the institution's network (d). A network firewall (e) is used both to protect the broader network community from accidental or malicious actions within the virtual ne twork and to restrict access to the virtual network for only those students enrolled in the subject. The firewall is configured to only allow RDD sessions. Any configuration proble ms either with the virtual network or the host computer will be confined to the teaching network (c). 


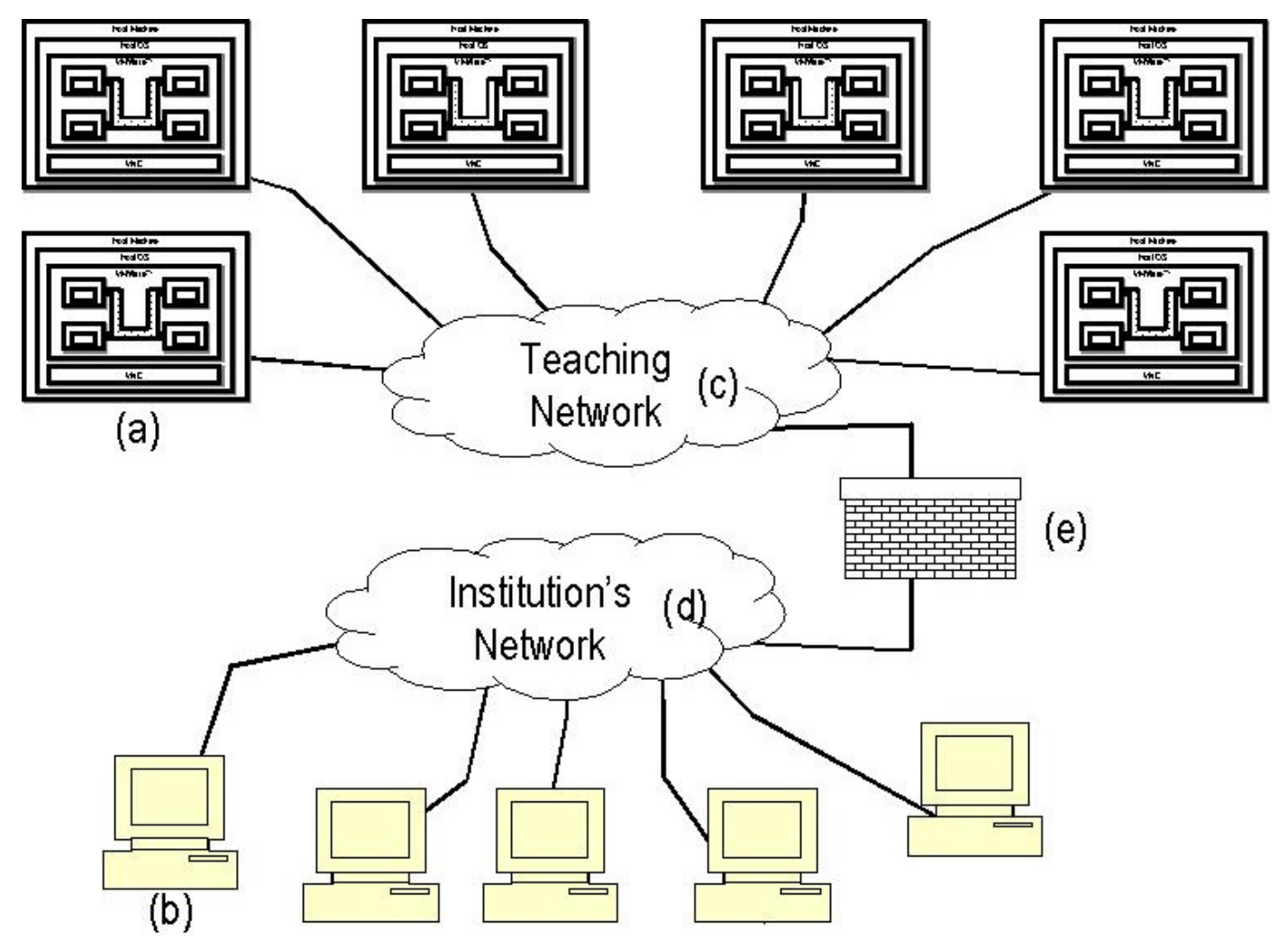

Figure 4. Typical remote access session to Velnet.

The first experiment with Velnet restricted access to client computers within the institution. Once security issues for wider Internet access are resolved, it would be possible for students to connect from anywhere using a web browser.

\section{Pilot Study}

\section{Aim}

The aim of the pilot study was to gain feedback from students and tutors as to whether Velnet might provide an enhanced learning environment for students studying computer networking.

\section{Velnet Configuration}

The pilot study consisted of six host machines running Windows $®$ XP, each with three virtual machines. One of the virtual machines ran OpenBSD while the other two virtual machines ran Windows ${ }^{\circledR}$ NT Workstation. Windows ${ }^{\circledR}$ XP remote desktop tools were used to provide students with remote connectivity.

\section{Participants}

The participants were students or former students. All had networking experience, such as two undergraduate courses and some home and small enterprise network establishment experience. The participants were new to Velnet. We intended to ask the participants to evaluate the usefulness of Velnet as a learning tool so sought more experienced and knowledgeable participants. We did not want the partic ipants to struggle with the lesson objectives, as this might have detracted from their ability to evaluate Velnet. 


\section{Lesson Objectives}

The lesson objective for the pilot study was to configure two private IP networks connecting to the existing School network, shown in figure 5. The first virtual machine (VM1) is constructed using three ne twork interfaces and OpenBSD configured to route IP packets between the interfaces.

The second and third virtual machines (VM2 and VM3) were each configured with one network interface and Windows ${ }^{\circledR}$ NT to act as clients on each of the private IP subnets.

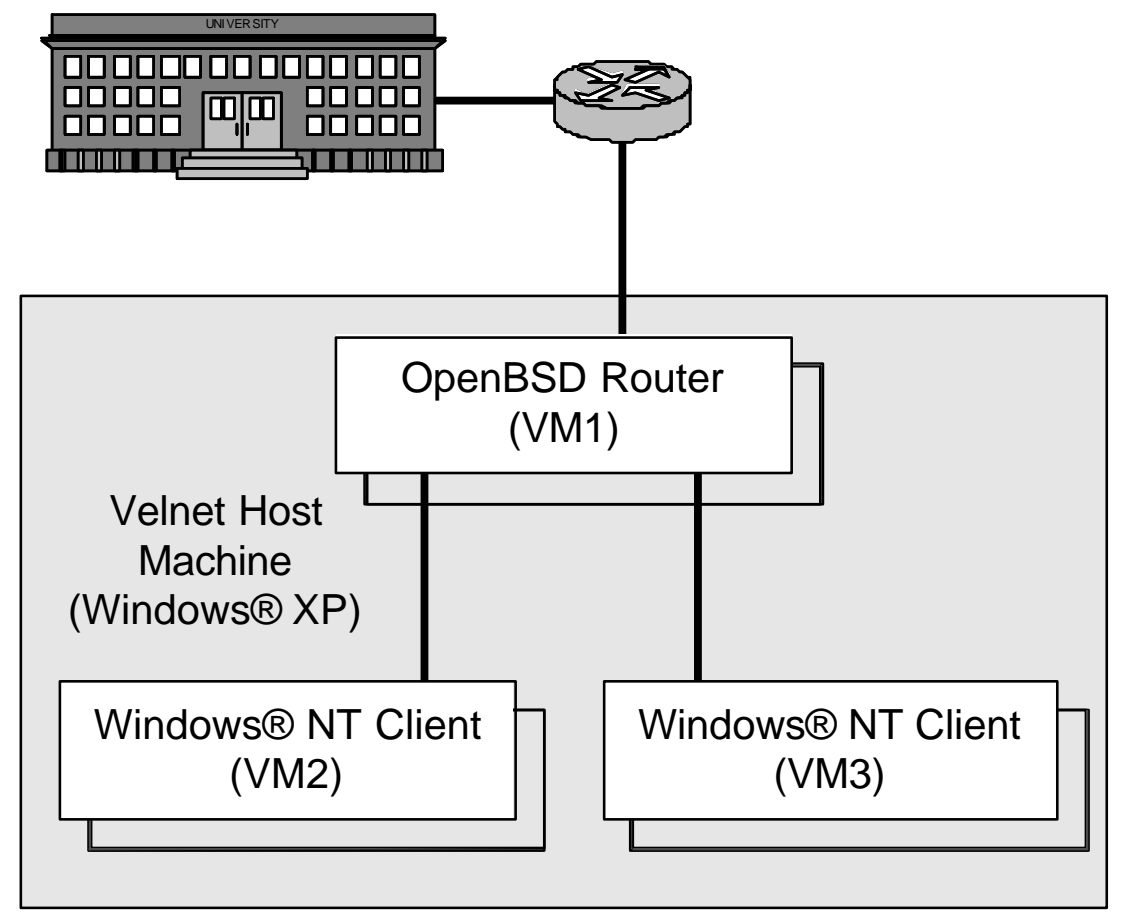

Figure 5. Lesson objective for the pilot study.

\section{Results}

Before commencing working with Velnet, participants were asked to write down their expectations of the environment. At the conclusion of the lesson, the participants were asked 11 open questions about what they thought of Velnet.

When asked if they considered Velnet an authentic learning environment the responses included "very realistic", "fairly authentic", and "8 out of 10".

The ease of use was considered less complex than some of the games the participants played. There was some concern about the cluttering of windows on the desktop, but this was considered no different to running several applications on a desktop.

The criticisms of the virtual verses a real environment were: that the virtual environment took away the idea of physical connectivity, and that understanding their own environment was "okay", but the instructors environment was confusing when they missed the instructors demonstration.

Asked whether they were conscious if the environment was virtual, the response was affirmative, particularly demonstrated by the difference in mouse behaviour.

All participants had their expectations met.

The participants recognise that the capability of Velnet could be beyond networking into other disciplines outside computing as well as other fields within computing such as programming. 
For the lesson that was undertaken during the pilot study the participants considered that Velnet was robust and secure. However, all noted that this was not thoroughly tested during the pilot and more work was required to be sure.

All participants recognised that Velnet's as cost-effective means of delivering an authentic learning environment.

The participants were asked what they understood the lesson to be and to describe the objective as a block diagram. One participant thought the virtual environment was part of the lesson, all others recognised the distinction between the lesson and using Velnet. All block diagrams were an approximation of that shown in figure 5 .

\section{Discussion}

The participants' responses indicated that Velnet is a viable learning environment. We are encouraged to continue with its use.

Our next study would need to include participants without networking experience to establish if the experience of the participants made their understanding of Velnet easier.

An important consideration is that Velnet is a learning environment; it is not the lesson itself. Care needs to be taken with the development of the supporting courseware so that focus is on course content rather than Velnet.

\section{Conclusion}

Velnet, a virtual environment for learning networking, is a significant resource that supports the "practical" application of computer networking knowledge and skills.

We solved most of our problems of providing a "hands-on", authentic learning environment by developing Velnet. We chose to use Velnet, as it is cheaper, it takes up less space; it is safer and more manageable than an equivalent physical environment. We described the design of Velnet as one or more host machines and operating systems, commercial virtual machine software, virtual machines and their operating systems, a virtual network connecting the virtual machines, and remote desktop display software. At our institution, our first experiment with Velnet used existing hardware and software on other standalone machine. The pilot study allowed participants to connect to Velnet via our institution's network.

Difficulties prevented the use of VNC during the pilot, VNC is still considered to be viable in the long term due to its open source licensing and its availability for numerous computing platforms. Further investigation is needed into the way VNC and VMWare interact to overcome the difficulties.

Further evaluation of Velnet as a learning environment is our immediate research direction. A larger study with less experience participants is our next step. We also hope to establish if students do take advantage of the availability of Velnet to enhance their networking knowledge and consequently have improved learning experiences and outcomes.

Velnet is the application of a well-established practice of teaching by simulation to the teaching of computer networking. The extension of the virtual environment: into other topics; to the Internet; into virtual reality, and an articulate virtual laboratory are planned. In the short-term, we can use Velnet to teach networking, systems administration, network administration, security, and management, and Web administration and security. For the medium-term, we aim to provide these topics online by incorporating Velnet into new instructional and assessment designs. In addition, in the medium-term, we would like Velnet to be the base for a virtual reality overlay that can be used to explore concepts and non-detectable physical events that take place in a network and to slow down time. The long-term future of Velnet is likely to be directed at making it an articulate virtual laboratory. 


\section{References}

AGILENT TECHNOLOGIES. (2001). Agilent Technologies offers free network troubleshooting software to colleges and universities; Professional tool helps teach students modern communication protocols and networking principles. M2 Presswire, 1.

Asia Computer Weekly. (1999). Networld+Interop99(N+I99) PREVIEW: Sign up for the ATM Classroom at Fore University. Asia Computer Weekly, 1.

Biggs, J. (1999). Teaching for quality learning at university. Buckingham, England: Society for Research into Higher Education and Open University.

Braathen, S., \& Robles, M. (2000). The importance of assessment in business education. National Business Education Yearbook, 2000, 11-24.

Cane, A. (1999, Feb 25, 1999). Cisco to build national network for Telia NEWS DIGEST:; [London edition]. Financial Times, pp. 38.

Cisco. (2002, 16 November 2002). Academy Connection: Program Overview, [Web site]. Cisco Systems. Available: http://cisco.netacad.net/public/academy/About.html [2002, 25th November].

Clancey, W. J. (1993). Situated action: A neuropsychological interpretation: Response to Vera and Simon. Cognitive Science, 17, 87-116.

Connectix. (2002). Virtual PC ${ }^{\mathrm{TM}}$ for Windows User Guide (Second Edition). Connectix [2002, October 2002].

Costa, G. (2000). The Cisco certification programs. Exploring Windows NT, 8(4), 14.

Cruikshank, B. J. (2002). Can problem-based instruction stimulate higher order thinking? Journal of College Science Teaching [H.W. Wilson-EDUC], 31(6), 374.

Davis, N., Ransbottom, S., \& Hamilton, D. (1998). Teaching Computer Networks Through Modeling. ACM SIGAda Ada Letters, XVIII(5), 104 - 110.

Forbus, K. D., Whalley, P. B., Everett, J. O., Ureel, L., Brokowski, M., Baher, J., \& Kuehne, S. E. (1999). CyclePad: an articulate virtual laboratory for engineering thermodynamics. Artificial Intelligence [H.W. Wilson-AST], 114(1/2), 297.

Harrold, D. (2000). Virtual reality saves money! Control Engineering, 47(11), 36.

Kasim, S. (2002). More schools to offer ICT by 2005. Computimes Malaysia, 1.

Logical UK Ltd. (2002). Sheffield Hallam University awards network upgrade to Logical. M2 Presswire, 1.

Marti, W. F., Pooch, U. W., \& Hamilton, J. A. (1996). PACKET tracing: a new paradigm for teaching computer network courses. Paper presented at the ACM SIGCSE/SIGCUE Conference on Integrating Technology into Computer Science Education, Barcelona, Spain.

Mateyaschuk, J. (1999). Certification checks out for IT. Computer Reseller News(846), 52.

McInnis, C., \& Hartley, R. (2002). Managing Study and Work: the impact of full-time study and paid work on the undergraduate experience in Australian universities. Canberra: Department of Education, Science and Training.

Montgomery, K. (2002). Authentic tasks and rubrics: Going beyond traditional assessments in college teaching. College Teaching, 50(1), 34.

NORTEL NETWORKS. (2002). University of Miami to deploy next generation metro optical solution from Nortel Networks; Faster, more cost-effective transport network expected to enhance academic research. M2 Presswire, 1.

O'Donoghue, J., Singh, G., \& Dorward, L. (2001). Virtual education in universities: a technological imperative. British Journal of Educational Psychology, 32(5), 511-523.

Piccoli, G., Rami, A., \& Blake, I. (2001). Web-based virtual learning environments: A research frame work and a preliminary assessment of effectiveness in basic IT skills training. MIS Quarterly, 25(4), 401-426.

Richardson, T., Stafford-Fraser, Q., Wood, K. R., \& Hopper, A. (1998). Virtual Network Computing. IEEE Internet Computing, 2(1), 33-38.

Virtual PC ${ }^{\mathrm{TM}}$. (2002). Virtual PC ${ }^{\mathrm{TM}}$ (Version 5.1) [Provides virtual x86 machines]. San Mateo, California, USA: Connectix Corporation. 
VMWare ${ }^{\mathrm{TM}}$. (2002). VMWare ${ }^{\mathrm{TM}}$ Workstation 3.2 User's Manual. VMWare Inc. Available: http://vmwaresvca.www.conxion.com/software/ws32_manual.pdf [2002, October, 2002].

VMWare ${ }^{\mathrm{TM}}$ Workstation. (2002). VMWare ${ }^{\mathrm{TM}}$ Workstation (Version 3.2) [Provides virtual $\mathrm{x} 86$ machines]. Palo Alto, California, USA: VMWare Inc.

Winn, W. D., \& Jackson, R. (1999). Fourteen propositions about educational uses of virtual reality. Educational Technology [H.W. Wilson-EDUC], 39(4), 5-14.

\section{Biographies}

Ilona Box is a lecturer in the School of Computing and IT, University of Western Sydney. Her primary teaching areas are object-oriented analysis and design. Her research interests are information systems development and education, and improving the learning experience for all types of students. Her interest in the work in this paper is in the development of simulated and articulate virtual learning environments. She has published a textbook (Object-Oriented Software Development: Step by Step) in response to the need for a text targeted at students entirely new to object-oriented software development.

Bruce Kneale is an associate lecturer in the School of Computing and IT, University of Western Sydney. His primary teaching areas are computer networking and operating systems. His research interests are in cluster computing, data link layer communications and computer networking for health services. His interest in this paper is the development of virtual environments for teaching computer networking. 